\title{
Noise-Induced Resonance in Delayed Feedback Systems
}

\author{
C. Masoller \\ Instituto de Física, Facultad de Ciencias, Universidad de la República, Igua 4225, Montevideo 11400, Uruguay
}

(Received 23 January 2001; published 3 January 2002)

\begin{abstract}
We study the influence of noise in the dynamics of a laser with optical feedback. For appropriate choices of the feedback parameters, several attractors coexist, and large enough noise induces jumps among the attractors. Based on the residence times probability density, it is shown that with increasing noise the dynamics of attractor jumping exhibits a resonant behavior, which is due to the interplay of noise and delayed feedback. It is also shown that this type of resonance is not specific to the model equations used, since it also occurs in other delay differential equations.
\end{abstract}

DOI: $10.1103 /$ PhysRevLett.88.034102

It is well known that an adequate amount of noise can increase the order in the dynamics of a nonlinear system. Examples of the positive role of noise are the enhancement of the response of a bistable system to a weak periodic forcing signal (stochastic resonance) [1,2], the appearance of regular pulses in an excitable system ( $\mathrm{co}$ herence resonance) $[3,4]$, and others [5,6]. Most of the studies on noise-induced effects have been performed on systems that possess a small number of coexisting attractors, typically bistable systems. Arecchi and co-workers [7] have shown that the coexistence of basins of attraction in phase space may lead to the appearance of $1 / f$ noise. Systems that exhibit a large number of attractors lead to additional interesting phenomena, such as noiseinduced preference of attractor [8] and noise-enhanced multistability [9].

In this Letter, the effect of noise is studied in a multistable system, which is a single-mode semiconductor laser with weak optical feedback. Feudel and Grebogi have shown that chaotic attractors are uncommon in multistable systems [10]. However, optical feedback renders the laser a time-delayed system, which is an infinite-dimensional system, and allows for multistability and complex dynamics. Aside from the interest from the nonlinear dynamics point of view, semiconductor lasers are key elements in optical communication systems (where some amount of external feedback and noise are often unavoidable) and have been extensively studied both numerically and experimentally [11-13].

Without noise the system has several coexisting attractors, and large enough noise induces jumps from one attractor to another. It is shown that with increasing noise the dynamics of attractor jumping exhibits a resonant behavior, which is due to the interplay of noise and delayed feedback. The residence times probability density, $P(I)$, exhibits a structure of peaks centered at multiples of the external cavity round-trip time, superimposed on an exponentially decaying background. The background corresponds to jumps induced at random by the noise, while the peaks indicate resonance of noise and feedback. A fluctuation strong enough to induce a jump, due to the
PACS numbers: 05.45.Gg, 05.40.Ca, 42.65.Pc, 42.65.Sf

feedback, is reinjected into the system and might induce other jumps.

A structure of peaks in $P(I)$ (superimposed on an exponential background) has been observed in stochastic resonance $[2,14]$, with the peaks centered at $I \sim(n-1 / 2) T_{o}$ (where $T_{o}$ is the period of the forcing signal). Since the peaks signature synchronization of the noise-induced jumps to the external periodic forcing signal, a measure of the resonance is given by the strength of the peaks. Here, we adopt this definition to measure the resonant behavior, and it is shown that the strength of the $n$th peak reaches a maximum for an optimal level of noise. It is also shown that this type of resonance is not specific to the model equations used, since it also occurs in other delayed feedback equations.

The Lang-Kobayashi model for a single-mode semiconductor laser with weak optical feedback is [15]

$$
\begin{gathered}
\dot{E}=k(1+i \alpha)[G-1] E+\gamma E(t-\tau) e^{-i \omega_{o} \tau} \\
+\sqrt{D} \xi, \\
\dot{N}=\left(j-N-G|E|^{2}\right) / \tau_{n} .
\end{gathered}
$$

Here, $E$ is the slowly varying complex field, and $N$ is the normalized carrier density. The parameters are $k$ is the cavity losses, $\alpha$ is the linewidth enhancement factor, $G=N /\left(1+\epsilon|E|^{2}\right)$ is the optical gain (where $\epsilon$ is the gain saturation coefficient), $\gamma$ is the feedback intensity, $\omega_{o}$ is the optical frequency without feedback, and $\tau$ is the round-trip time in the external cavity. $\xi(t)$ is a complex Gaussian white noise, and $D$ measures the noise intensity. $j$ is the normalized injection current, and $\tau_{n}$ is the carrier lifetime.

The attractors of the deterministic model were studied in detail in [16]. Without feedback there is only one fixed point, which corresponds to the emission mode of the solitary laser. Weak feedback introduces new fixed points, and for increasing $\gamma$, a chaotic attractor develops from each initially stable fixed point. The route to chaos is a quasiperiodic route, and the two frequencies that appear are the relaxation oscillation frequency and the external cavity frequency (which is nearly equal to $1 / \tau$ ). 
For appropriate choices of $\gamma$ and $\tau$, several stable chaotic attractors coexist; five of them are shown in Fig. 1(a). They are plotted in the plane formed by $\left(\Delta \phi,|E|^{2}\right)$, where $\Delta \phi=\phi(t)-\phi(t-\tau)$. The dynamics in an attractor has two stages of motion [see Figs. 1(b) and 1(c)]. The trajectory spends some time orbiting around the destabilized fixed point, until it reaches the neighborhood of the fixed point. Then, the trajectory traverses the vicinity of the fixed point and starts oscillating again. This process, in which the trajectory is orbiting around the fixed point, or is in the vicinity of the fixed point, keeps repeating with a period nearly equal to $\tau$ [17].

Large enough noise induces jumps from one attractor to another. The attractor jumping is not observed on the time evolution of the laser intensity [Fig. 1(d)] but is clearly distinguished on the time evolution of the phase delay [Fig. 1(e)]. The effect of noise on a stochastic trajectory is shown on the left column of Fig. 2. For low noise [Fig. 2(a)], the trajectory might spend a large amount of time in an attractor before noise induces a jump. Larger noise levels induce more frequent jumps [Figs. 2(b) and 2(c)], and the mean residence time on an attractor diminishes. For even larger noise [Figs. 2(d) and 2(e)], the dynamics becomes increasingly noisy, until there is almost no structure present in the trajectory.

Next, the statistics of the residence time in an attractor is investigated as a function of the noise intensity. The time interval between two consecutive jumps was determined by approximating the jumps as instantaneous events. This approximation is good if the noise is not too large. For large noise, the jumps are too frequent to accurately determine the jumps. For even larger noise, the stochasticity is dominant, and the attractor jumps lose physical meaning.

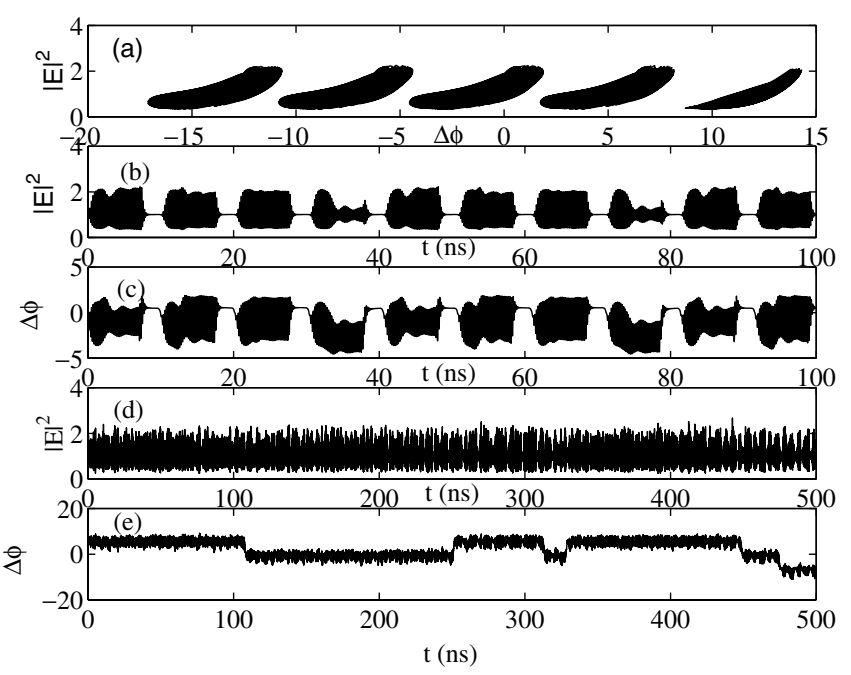

FIG. 1. (a) Five coexisting attractors when $D=0$, and $k=$ $500 \mathrm{~ns}^{-1}, \epsilon=0.003, \tau_{n}=1 \mathrm{~ns}, \alpha=3, j=2, \omega_{o} \tau=6 \mathrm{rad}$, $\gamma=2 \mathrm{~ns}^{-1}$, and $\tau=10 \mathrm{~ns}$. Time evolution of the intensity (b), and the phase delay (c), when the trajectory evolves on an attractor. Time evolution of the intensity (d), and the phase delay (e), when $D=0.0225 \mathrm{~ns}^{-1}$.
The center column of Fig. 2 shows the residence times probability distribution, $P(I)$, on a logarithmic scale. For large residence times, $P(I)$ exhibits a negative exponential behavior. For short residence times, $P(I)$ exhibits a structure of peaks (shown in the right column of Fig. 2) that will be discussed later. Notice that for low noise there is a large dispersion in the values of $I$ that decreases with increasing noise.

Figure 3(a) shows the standard deviation of the normalized residence time on an attractor, $R=\operatorname{std}(I /\langle I\rangle)$. $R$ exhibits a minimum at an intermediate noise intensity $\left(D \sim 0.01-0.02 \mathrm{~ns}^{-1}\right)$, which characterizes coherence resonance behavior [3,4]. The minimum can be understood as follows. For low noise, there is a large dispersion of the values of $I /\langle I\rangle$, and the value of $R$ is large. For intermediate values of the noise intensity, noise triggers more frequent jumps and the dispersion of the values of $I /\langle I\rangle$ decreases. For larger noise, the dynamics becomes increasingly irregular, and the dispersion of the values of $I /\langle I\rangle$ increases. For even larger noise, the jumps are so frequent that the dispersion of the values of $I /\langle I\rangle$ decreases again.

A minimum of $R$ for an optimal level of noise was first reported by Pikovsky and Kurths [3], studying the dynamics of the excitable Fitz-Hugh-Nagumo system under external noisy driving. In [3] the minimum is due to the different effect of noise on the duration of the two stages of motion of the dynamics. One stage is the activation time, which corresponds to evolution in the neighborhood of the fixed point, and the other stage is the excursion time, which corresponds to evolution along the homoclinic orbit that connects the fixed point with itself. Here, the different effect of noise on the residence time on an attractor, and
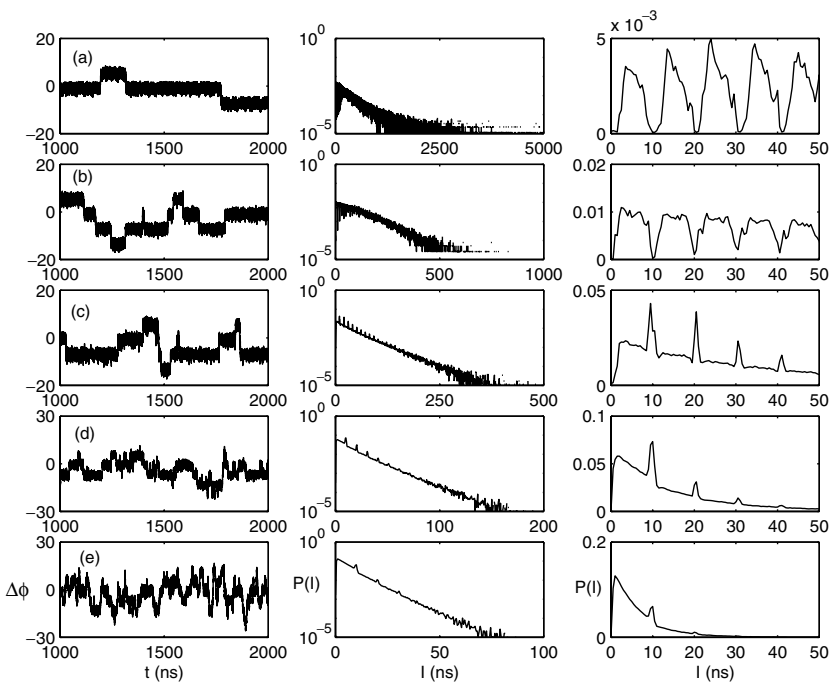

FIG. 2. Left column: five stochastic trajectories for increasing noise levels. Center column: corresponding $P(I)$ on a logarithmic scale. Right column: $P(I)$ for short residence time on a linear scale. (a) $D=0.0025 \mathrm{~ns}^{-1}$, (b) $D=0.0225 \mathrm{~ns}^{-1}$, (c) $D=0.09 \mathrm{~ns}^{-1}, \quad$ (d) $D=0.16 \mathrm{~ns}^{-1}, \quad$ (e) $D=0.25 \mathrm{~ns}^{-1}$. All other parameters are as in Fig. 1. 

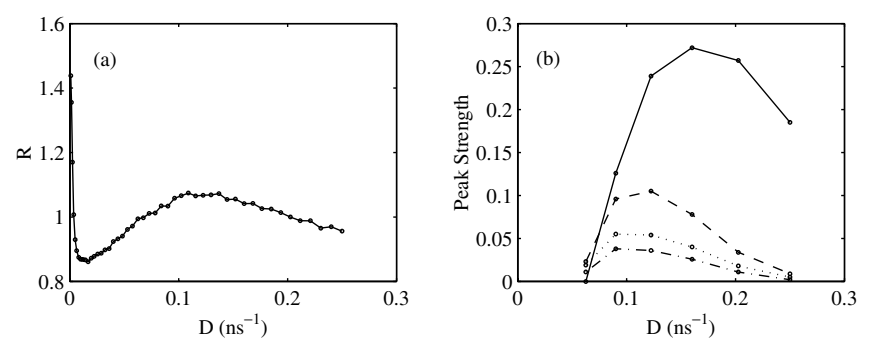

FIG. 3. (a) Standard deviation, $R$, of the normalized residence time on an attractor, as a function of the noise intensity. The results shown are evaluated from samples of $5 \times 10^{5}$ jumps. (b) Peak strengths $P_{1}$ (solid line), $P_{2}$ (dashed line), $P_{3}$ (dotted line), and $P_{4}$ (dash-dotted line), as a function of $D$. To accurately subtract the exponential background, a very high statistics was needed (the results shown are evaluated from samples of $3.5 \times 10^{6}$ jumps). The parameters are as in Fig. 1 .

on the jumping time, leads to a minimum of $R$. However, there is a new mechanism leading to resonant behavior, which is uncovered by the probability distribution of short residence times.

For short $I, P(I)$ (right column in Fig. 2) exhibits a structure of peaks, which depends on the noise intensity. On increasing the noise level from small up to large values, $P(I)$ goes from a multipeaked distribution with minimums (or "gaps") for values of $I$ that are multiples of $\tau$ to a multipeaked distribution with maximums for values of $I$ that are multiple of $\tau$. The multipeaked distribution is superimposed onto an exponentially decaying background, which is weak for low noise but that grows and hides the peak structure for large noise.

For low noise, the gaps of $P(I)$ at $I \sim n \tau$ can be understood by considering the moment when the trajectory can jump from one attractor to another. The jump to an attractor with lower $\Delta \phi$ usually occurs in the middle of the stage in which the trajectory oscillates around the fixed point, while the jump to an attractor with larger $\Delta \phi$ occurs at the end of the oscillating stage. Therefore, for low noise, residence times that are multiples of $\tau$ are less probable. For larger noise, the deterministic dynamics on an attractor is mostly washed out by the noise, and a jump can occur at any time. For even larger noise, the residence times $I \sim n \tau$ become increasingly probable. This unexpected feature is caused by the delayed feedback and can be understood in the following terms: a fluctuation strong enough to trigger a jump, due to the delayed feedback term, might induce another jump, one or a few delay times later.

Following the discussion of [2,14], the strength of the $n$th peak, $P_{n}$, is characterized by the area under the peak, once the background (that corresponds to jumps induced at random by the noise) was subtracted. Figure 3(b) shows $P_{n}$ as a function of $D$, for the first four peaks. As in [2,14], each curve passes through a maximum, and the noise level at which the maximum occurs decreases with the index $n$. This is due to the fact that $\langle I\rangle$ diminishes with increasing noise, and the trajectory does not stay in an attractor long enough to jump out of it due to the delayed effect of the fluctuation that induced the original jump.
No significant differences are found when the strength of a peak is measured by its height (after background subtraction). However, it is worth mentioning that for large noise the background has a slightly different decay rate on the left side than on the right side of a peak (in this case, to subtract the background, an average decay rate was used). This feature deserves further investigation, since it also occurs when the noise-induced jumps are among fixed points (for lower $\gamma$ ). In this case, the mean residence time depends on the noise level according to Kramers law [17], $\langle I\rangle \sim \exp [U / D]$ (where $U$ is a constant), in spite of the fact that $P(I)$ exhibits peaks for $I \sim n \tau$ within a "discontinuous" exponentially decaying background. Similar results were reported in [18], in a model with two states, where the transition probability depends on the state at $\tau$ steps past. In [18], $P(I)$ exhibits a single peak at $I \sim \tau$ and a different dependence on $I$ if $I<\tau$ or $I>\tau$.

One might wonder if the resonance found is not a bona fide resonance [2,14], in the sense that the strength of the peaks might achieve a maximum value by varying a parameter while keeping the noise level fixed. However, varying the external cavity parameters does not lead to resonant behavior. Increasing the feedback (but keeping it lower that the feedback level such that the coexisting attractors merge into a global attractor) leads to an increase of the strength of the peaks. This can be understood by noticing that when $\gamma$ increases, the delayed term increases its strength, and, therefore, the probability of inducing additional jumps increases. On the contrary, when $\tau$ increases, the strength of the peaks diminishes. This in due to the fact that $\langle I\rangle$ is nearly independent of $\tau$, and, therefore, as $\tau$ increases, the system does not stay on an attractor long enough to jump out of it induced by the delayed effect of the fluctuation that originated the initial jump.

Next, we show that the resonance found is not specific of the model equations used. We consider a simple equation that was originally derived for the evolution of the phase of the optical field, when the fluctuations of the field amplitude are small [19]:

$$
\dot{\phi}=a \sin [b+\phi(t)-\phi(t-\tau)]+\sqrt{D} \xi(t) .
$$

Here, $a$, and $b$ are parameters, $\tau$ is the delay time, $D$ is the noise intensity, and $\xi(t)$ is a Gaussian white noise. Figure 4(a) shows a typical stochastic trajectory, in which there are noise-induced jumps among fixed points [such that $\left.\phi(t)=\omega_{i} t\right]$, and Fig. 4(b) shows the corresponding residence times probability density. Peaks at multiples of $\tau$ are clearly observed. Figure 4(c) shows the mean residence time, as a function of noise, which verifies Kramers law, and Fig. 4(d) shows that the height of the peaks present a resonant behavior for increasing noise.

Finally, we discuss the effect on a more general context. We consider the equation

$$
\dot{x}=x(t)-x(t-\tau)-[x(t)-x(t-\tau)]^{3}+\sqrt{D} \xi,
$$



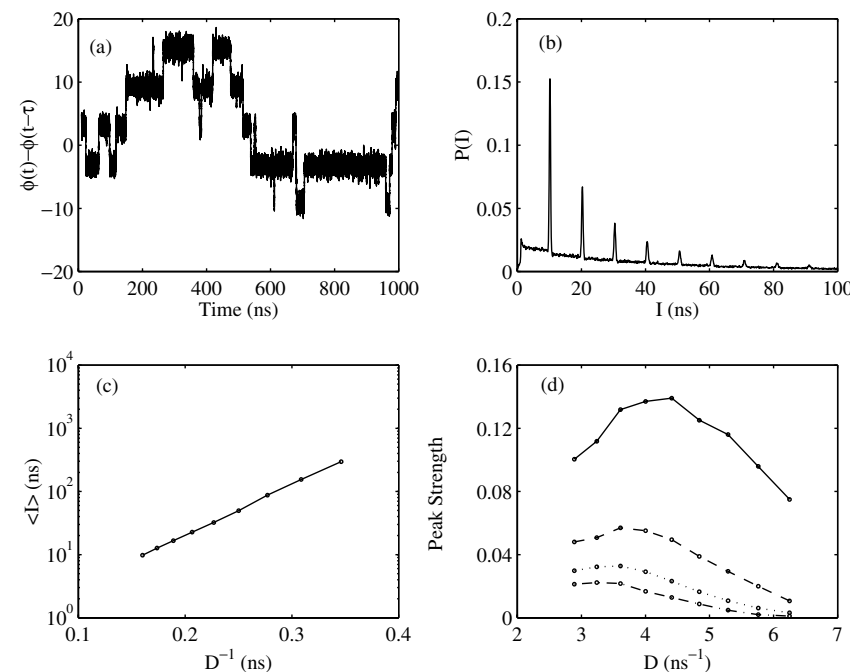

FIG. 4. (a) Numerical simulation of Eq. (3) with parameters $a=10 \mathrm{~ns}^{-1}, \quad b=0.1 \mathrm{rad}, \quad \tau=10 \mathrm{~ns}, \quad$ and $D=4 \mathrm{~ns}^{-1}$. (b) Residence times probability density. (c) Mean residence time, as a function of $D^{-1}$. (d) Height of the first four peaks, as a function of $D$.

which has steady-state solutions of the form $x(t)=x_{s} t$ with $x_{s}=0, x_{s}= \pm \sqrt{1-1 / \tau}$. Figure 5 shows that peaks appear in the histogram of residence times, which are also spaced by the delay time.

In conclusion, the effect of noise was studied in a system with a delayed feedback mechanism, and the major finding of the analysis is a resonant behavior in the noise-induced attractor jumps, which is due to the delayed feedback. The residence times probability density exhibits a structure of peaks at multiples of the delay time, and the strength of the peaks reaches a maximum for an optimal level of noise. We have presented a qualitative explanation of the effect. A detailed theoretical analysis is in progress and will be reported elsewhere.

Feedback systems appear in many relevant fields of physics, biology, and electronics. In many systems the feedback mechanism stabilizes and controls the dynamics,

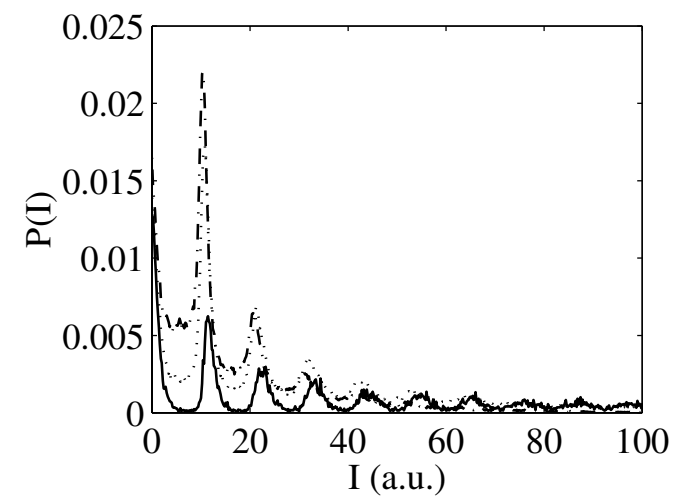

FIG. 5. Residence times probability density obtained from simulations of Eq. (4) with $\tau=10$ and $D=0.05$ (solid line), $D=0.10$ (dotted line), and $D=0.15$ (dashed line). but in other systems, the feedback destabilizes and induces chaotic oscillations. Our results suggest that in this latter type of system, interesting phenomena might appear due to the interplay of noise and delayed feedback.

The author acknowledges stimulating discussions with M. G. Rosenblum and thanks P. Hänggi for the possibility of presenting this work at the Workshop on Physics of Information and Synchronization in Stochastic Dynamics, in Dresden, Germany. This work was supported in part by CSIC and PEDECIBA.

[1] L. Gammaitoni, P. Hänggi, P. Jung, and F. Marchesoni, Rev. Mod. Phys. 70, 223 (1998).

[2] L. Gammaitoni et al., Phys. Rev. Lett. 74, 1052 (1995); M. H. Choi, R. F. Fox, and P. Jung, Phys. Rev. E 57, 6335 (1998); F. Marchesoni et al., Phys. Rev. E 62, 146 (2000).

[3] A. S. Pikovsky and J. Kurths, Phys. Rev. Lett. 78, 775 (1997).

[4] J. R. Pradines et al., Phys. Rev. E 60, 6407 (1999); S. K. Han et al., Phys. Rev. Lett. 83, 1771 (1999); B. Lindner and L. Schimansky-Geier, Phys. Rev. E 61, 6103 (2000); C. Palenzuela et al., e-print cond-mat/0007371.

[5] M. Santagiustina et al., Phys. Rev. Lett. 79, 3633 (1997); Phys. Rev. E 58, 3843 (1998); H. Hempel et al., Phys. Rev. Lett. 82, 3713 (1999).

[6] M. San Miguel and R. Toral, Instabilities and Nonequilibrium Structures VI, edited by E. Tirapegui, J. Martínez, and R. Tiemann (Kluwer Academic Publishers, Dordrecht, The Netherlands, 2000), pp. 35-130.

[7] F. T. Arecchi and F. Lisi, Phys. Rev. Lett. 49, 94 (1982); F. T. Arecchi et al., Phys. Rev. Lett. 49, 1217 (1982); F. T. Arecchi, Chaos 1, 357 (1991).

[8] K. Kaneko, Phys. Rev. Lett. 78, 2736 (1997); , Physica (Amsterdam) 124D, 322 (1998); S. Kraut, U. Feudel, and C. Grebogi, Phys. Rev. E 59, 5253 (1999).

[9] S. Kim et al., Phys. Rev. Lett. 78, 1616 (1997).

[10] U. Feudel and C. Grebogi, Chaos 7, 597 (1997).

[11] I. Fischer, G. H. M. van Tartwijk, A. M. Levine, W. Elsässer, E. Göbel, and D. Lenstra, Phys. Rev. Lett. 76, 220 (1996); G. Vaschenko et al., Phys. Rev. Lett. 81, 5536 (1998); J. Hales et al., Phys. Rev. Lett. 85, 78 (2000); E. A. Viktorov and P. Mandel, Phys. Rev. Lett. 85, 3157 (2000); Jing-Yuan Ko et al., Phys. Rev. Lett. 86, 4025 (2001).

[12] G. Giacomelli, M. Giudici, S. Balle, and J. R. Tredicce, Phys. Rev. Lett. 84, 3298 (2000).

[13] J. M. Buldu et al., e-print nlin.AO/0104047.

[14] G. Giacomelli et al., Phys. Rev. Lett. 82, 675 (1999); S. Barbay et al., Phys. Rev. E 61, 157 (2000).

[15] R. Lang and K. Kobayashi, IEEE J. Quantum Electron. 16, 347 (1980).

[16] C. Masoller and N.B. Abraham, Phys. Rev. A 57, 1313 (1998).

[17] H. A. Kramers, Physica (Utrecht) 7, 284 (1940).

[18] T. Ohira and Y. Sato, Phys. Rev. Lett. 82, 2811 (1999); T. Ohira and T. Yamane, Phys. Rev. E 61, 1247 (2000).

[19] J. Mörk, M. Semkow, and B. Tromborg, Electron. Lett. 26, 609 (1990); D. Lenstra, Opt. Commun. 81, 209 (1991). 